\title{
Integrated Skills, Integrated Data: Mapping Best Practice and Collections for Innovation and Engagement
}

\author{
Rachel Hosker, Claire Knowles, Norman Rodger \\ University of Edinburgh, Library and University Collections \\ ${ }^{\text {a) Corresponding author: Rachel.Hosker@ed.ac.uk }}$ \\ ${ }^{b)}$ Claire.Knowles@ed.ac.uk \\ c)
}

\begin{abstract}
In recent years the University of Edinburgh [UoE] has seen change, mergers, external partnerships and innovation at the heart of its growth and activity. Collections at UoE were not immune from these changes and have pioneered projects that both support and highlight unique educational cultures. Technology and the dissemination of collections has not only engendered positive relationships with academics but has created wider opportunities for the use of collections in teaching, learning and research. This momentum and an established commitment to the interoperability of data and standards presented an opportunity to look for a global solution to collections management within the converged, cross disciplinary environment. This included harnessing expertise in the University with systems development for large European projects and wider project management. This session will explore how UoE became the first European contributor to the collections management tool Archives Space. Snapshots of a converged culture and how 'archives' have benefited from this (including how 'techies' and 'archivists' worked together). An upbeat finale will look at what the team at the UoE achieved and are excited about for the future.
\end{abstract}

\section{INTRODUCTION}

The development of best practice in managing collections data, collections related projects and people at the University of Edinburgh demonstrates an innovative approach to how all of these need to be identified and mapped. There are clear benefits of doing this within a converged culture of professional archivists, curators, librarians, project managers and software developers. High level skill sets for data management within teams housed in Universities are often seen, however the most successful output can be difficult to achieve when too much focus is placed on separate professional disciplines. This can stifle effective communication and collaboration for project work. The linking of complementary collections management thought (archives, libraries, and museums science) and technical expertise of software development teams with overarching project managers aware of the cultural needs and requirements of collections and their users has proved its worth. This is demonstrated through two case studies here and the successful launch of a number of projects using and making collections and collections data available in a creative way at the University of Edinburgh. The contextual background to these developments includes working in an ambitious world class university which has collections of world class importance. These collections relate directly to high-level academic research with impact on our understanding of health, welfare, culture, society, and education. This provides a requirement to look outside our own institution and adopt an open, flexible approach to working in collaboration and exploiting opportunities for the advantage of the University, collections, education and research. 
There are a number of national and international institutions and project comparators and collaborators that we became involved with. This allowed us to have external checks and balances and prevent too much of an internal focus. The University of Edinburgh has led Musical Instruments Museums Online (MIMO) [1] and Europeana [2] projects and work and been proactive members of UK The Archives Hub portal [3] and ArchivesSpace [4] with information from this feeding through to Europeana and the Archives Portal Europe [5].

\section{CULTURE SHIFT: MAKING BEST USE OF EXPERTISE AND SKILLS}

A restructure of the Library and University Collections Division (L\&UC) at the University of Edinburgh followed the appointment of a new Director of Library and University Collections in 2012, and led to four sections being formed (Research and Learning Services, Collections Development and Access, Special Collections and Centre for Research Collections, and Museums).[6] The four heads of sections along with the Director form the Senior Management Team. Out of this restructure came a vision of an ambitious and dynamic service with discovery of the collections at the heart of it.

'Library and University Collections (L\&UC) is responsible for the University of Edinburgh's digital and physical collections. We work in partnership to deliver the services that bring our collections to the university community and the wider public. L\&UC is a Division of Information Services working within a converged structure of IT, Library, Online Learning and Museums. The range of collections includes over 3,500,000 physical items, 330,000 ebooks, 50,000 journal titles, and 60KM of museum objects, archives, manuscripts and rare books. Fast, seamless discovery providing access to and use of the collections is vital, whether that be via an online device or physically, in one of our ten libraries or six museum facilities. Investment in the collections and services will continue to be made to ensure that current and future needs are met.

Vision: A library and university collection that is at the heart of education, research and engagement at Edinburgh, with an international reputation for innovation, quality and excellence.' [7]

Along with the formation of the four sections, four cross cutting groups were also formed which include team members from all of the sections. These cross cutting groups covered the themes of digital asset management, discovery, engagement and funding.

\section{Digital Asset Management Group (LAUC-DAM)}

This group was tasked with providing an overview to all projects and systems with digital asset management capability: whether holding scholarly materials, digital surrogates of special collections, or teaching materials. This included topics such as system architecture, digital preservation, presentation and delivery.

\section{Resource Description and Discovery Group (LAUC-DISCOVER)}

Recognising the direct link between resource description and discovery, this group was tasked with ensuring that the end-to-end relationship is taken into account throughout relevant systems and processes. This will help to ensure that our subscription, local, and unique contents are all described and discoverable in the best manner possible.

\section{Academic, Student, and Public Engagement Group (LAUC-ENGAGE)}

This group was tasked to develop initiatives which facilitate and encourage knowledge exchange, public engagement and promotion, and to provide a focus for incubating partnerships and collaborations which support the academic success of staff and students and contribute to user and visitor experience. 


\section{Projects and Fundraising Group (LAUC-FUND)}

This group was tasked with maximising our potential to enlarge financial support for L\&UC by providing an overview to all projects currently in development (not at delivery stage) and acting as a catalyst in incubating new projects. This included all potential income streams such as philanthropic giving (trusts, foundations individuals), grant awards and increased commercialisation.

With these areas of focus, the groups had some overarching considerations and aims. These are as follows:

Joining up across $L \& U C$-The nurturing of a culture of real, effective and mutually beneficial bridges across L\&UC was one of the main things we wanted to achieve and encourage. That we could help one another overcome obstacles by working together, technical development and input, metadata techniques and management, specialised curatorial and subject based knowledge combined effectively to deliver a common goal such as a specific resource discovery project, or better public engagement such as practical evening class workshops.

Strategic - The groups provided a chance for wider groups of L\&UC staff to feed in more effectively into strategic planning. The University's strategic plan [8] could sometimes seem very remote from what is actually happening on the ground. In essence it was to encourage new ideas and initiatives to drive the division forward through sharing them with these groups. Library and University Collections relies on its staff to keep abreast of developments in their field, and we were aware that in some areas we were leading the way amongst our peer institutions. It was felt that it was important for staff to feed into the development of our various professions.

Reflective - An important part of thought processes that produce new ideas and work is to reflect and check developments. Within the UK sector we were aware that celebrating success or good work was not as apparent as elsewhere in the sector worldwide. Sharing our achievements and accolades was another aim of these groups to help foster a sense of togetherness and pride, both about the University but also about our professional practice. By being reflective we were also realistic and talked about what was not working so well and what we could do to improve a project or service.

Analytical - It was important to adopt a scientific approach and dissect current procedures, work together in problem solving, especially if expertise from another part of the division could help resolve something or move it on to another stage of development. A culture of solution based outputs from these groups was established from the start.

As well as the wider L\&UC changes, there were many changes taking place within the Special Collections part of the University of Edinburgh. There had been major changes in the archival profession in terms of skills and the application of technology for discoverability in the early 2000s. Recognition of the successes of archival projects using professionally trained archivists allowed a bank of skills and knowledge to be built up within the University. This could also be seen in to be happening in the wider archival sector in Scotland. Within the University of Edinburgh the University Archivist had built up from a team of one professional archivist to a bank of two senior archivists to support the role, a professional conservator, and several project archivists. The archive collections were also being seen as requiring distinct professional treatment. This growth in people and skills was also reflected in the University Museums structure where further curators with specific expertise and skills, such as with musical instruments or conservation training were brought in. This led to a changes in working culture and practice with an enthusiasm for working cross-sectorally, feeding both the professional requirements and the user needs. The L\&UC Vision and Values statement that was developed and signed off in 2013 provides a focused look at that ethos. The core vision was to make all collections accessible and some of the most difficult to find were the archival collections.

As part of this changing culture it is useful to note the influence of the individual on working practice and culture within an organisation. Many cataloguing, intellectual arrangement and descriptive practices in use before standards evolved can be described as having the stamp of the individual on them with approaches that had an individual interpretation of what was required, without the guidance framework provided by standards. Standards and developing collaborative approaches to systems provide for a collective understanding and recognition to make them easier to communicate and have a broader sense of relevance. 
The following case studies demonstrate this collaborative approach using best practice and developing standards that informed the application of professional cataloguing and descriptive standards as well as wider user access and understanding of the collections.

\section{CASE STUDY 1: MUSICAL INSTRUMENT MUSEUMS}

\section{Discovering Hidden Collections: Musical Instruments Museums Online}

From 2009-2011, the University of Edinburgh was the lead partner in a major European funded project entitled MIMO, Musical Instrument Museums Online. The catalyst for this project was the recognition that there was no easy way to discover information about musical instruments held in public collections and so the aim was to create a single access point to digital content and information on the collections of musical instruments held in European museums. The University led on this project due to the exceptional and rare instrument collections it holds. Many of the instruments are still playable and through an established concert programme, St Cecilia's Hall at the University is the only place in the world where it is possible to hear 18th century music in an 18th century concert hall played on 18th century instruments. [9]

The MIMO project enabled the digital content of nine major musical instrument museums from across Europe to be harvested from their collection databases and made available to all, both through Europeana and via the MIMO website.

This resulted in MIMO having just over 46,000 instruments digitised, but in terms of images, the figure was much higher, with 80,557 digital images in total (i.e. including multiple views) and a smaller number of video and audio files.

The University of Edinburgh provided content for 4100 musical instruments, although only one image per item was submitted to MIMO many more were taken as were some video and audio recordings.

The project also successfully established the technical infrastructure that facilitates the harvesting of our museum content into MIMO and on to Europeana and, alongside that, the classification and thesauri that underpins multilingual searching.

Another key output was the production of a digital standards document, which set out to establish a global standard for the digitisation of musical instruments, with mandatory and recommended views, and tips and techniques for the photography of instruments. [10]

Since the end of the project the core partnership continued to develop the service and we currently have the content of twenty-two museums online, making us the world's biggest database of musical instruments held in public collections. The aim now is to attract other museums to add their collections to MIMO and to reach a point where MIMO can become the single access point for information on musical instrument collections for the entire world.

\section{Local Reflections on MIMO's Success}

Locally however, the end of the MIMO project brought into focus the inadequacies of our own system. As we did not have a fully searchable website for collections discovery at the University of Edinburgh anyone coming to our site was faced with seriously outdated provision which failed to make use of the technology and resources that had been developed for MIMO and Europeana.

Deficiencies in our existing site, along with a Repository Review Away Day, where we assessed our capability and provision for making our collections of born-digital and digitised items discoverable and accessible. This led to the decision to adopt a new repository infrastructure within $L \& U C$ to enable a more rapid release of digital content online within collections having their own branded web presence. Due to the knowledge and experience within the Library Digital Development Team for DSpace, it was decided to use this open source repository platform for the storage of digital items. DSpace has been in use at the University of Edinburgh since the Theses Alive! Project [11] in 2002-2004. [12] The Library Digital Development Team has a wealth of experience with DSpace, hosting repositories for six other Scottish Higher Education Institutions and a DSpace committer as a member of the team.

'The aim of the Repository Review is to establish a repository architecture for Library and University Collections, which allows collections to be ingested, managed and discovered in a robust extendable repository 
platform. This platform needs to be versatile, in order that it can be utilized by different collections, with differing needs, item and metadata types.' [13]

Items harvested for MIMO were already stored in a DSpace repository, which was populated from the Collections Management System Vernon (link). However, this site did not have the functionality we required, as there was limitations to searching and making changes to the site to make it obvious which collections were included and how to navigate through the content. This meant it was difficult to discover groups of instruments; title and headings were generic and not specific to the type of object; the images and audio-visual content could only be viewed through download; there was no collection branding or identity on the site, which diminished the collections web presence and ability to link the data about collections with related material in our collections and elsewhere.

The infrastructure adopted hides the DSpace repository using it for administrative functionality only and has a configurable user interface built in PHP on top of the Apache SOLR search [14] used by DSpace. The public user interface, Skylight [15], was developed by the University of Auckland, Library Applications, Development and Support team [16], who have assisted the development team in their adoption and customization of the Skylight application as used for http://collections.ed.ac.uk/mimed.

The use of SOLR provides search, faceted-search and browse functionality allowing users to quickly discover or drill-down search results. The SOLR configuration and website design was done by the Library developers in consultation with the Musical Instrument Curators, to ensure that it met the needs of the users and was tailored to the content. SOLR is configured to enable rapid discovery of an individual item for those who have a particular instrument in mind and also enable browsing for those who wish to view a subset of the collection.

Taking into consideration branding guidelines of the University of Edinburgh [17] and the desire to create a consistency between L\&UC collections websites and other sites for St Cecilia's the Musical Instrument Museum at the University of Edinburgh. This dictated the colour palette, font and use of the University logo. The design of the item pages was done in discussion with the curators who led on the ordering of fields, which metadata should be displayed, and the terminology to use. Musical instruments within the collection come in many shapes and sizes and this proved to be one of the greatest challenges with the design making the individual view for a clarinet as visually appealing as a harpsichord.

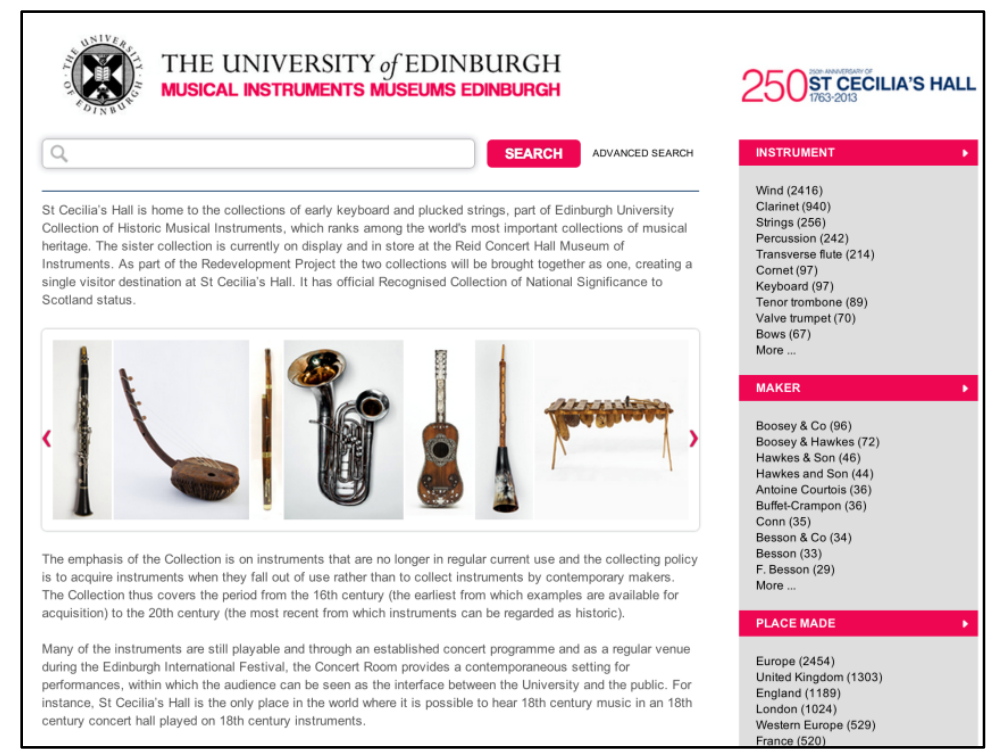

Figure1: Musical Instruments Museum at the University of Edinburgh Collections Home Page on Collections.ed.ac.uk/mimed. 


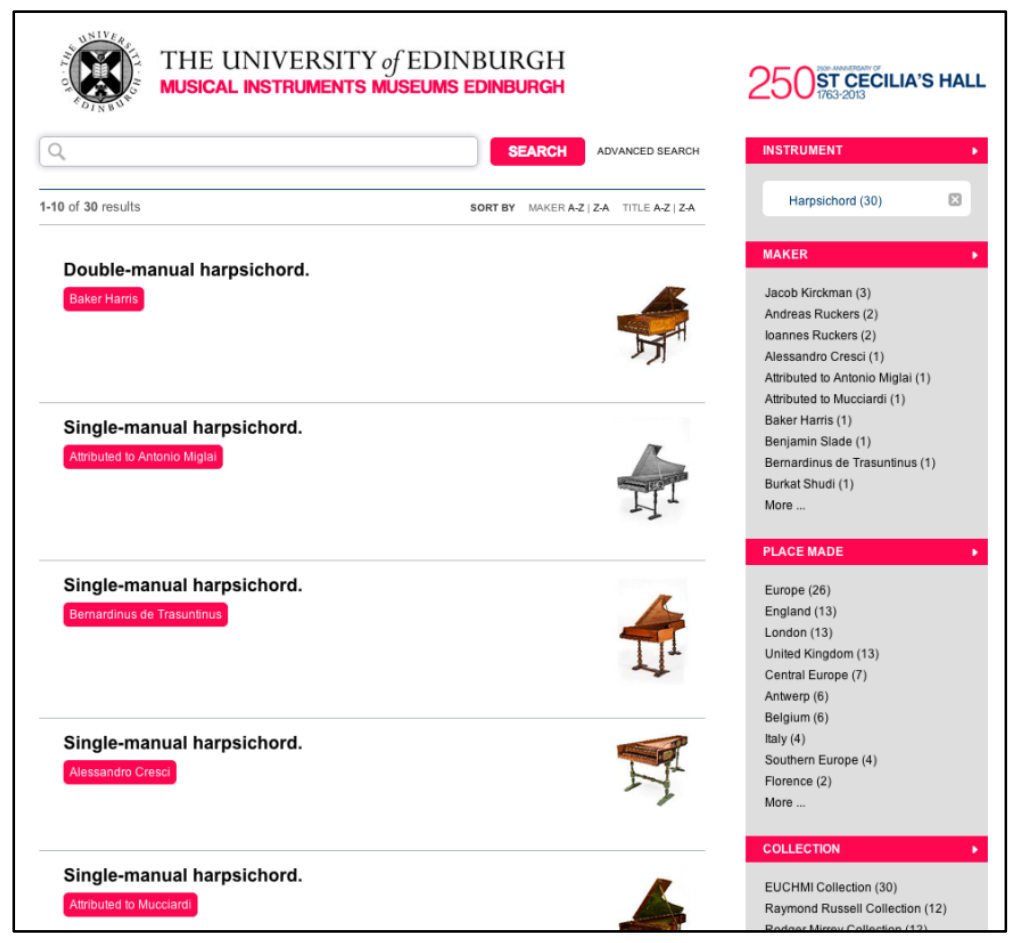

Figure 2: Display of search results showing the subset of a collection of harpsichords on collections.ed.ac.uk/mimed.

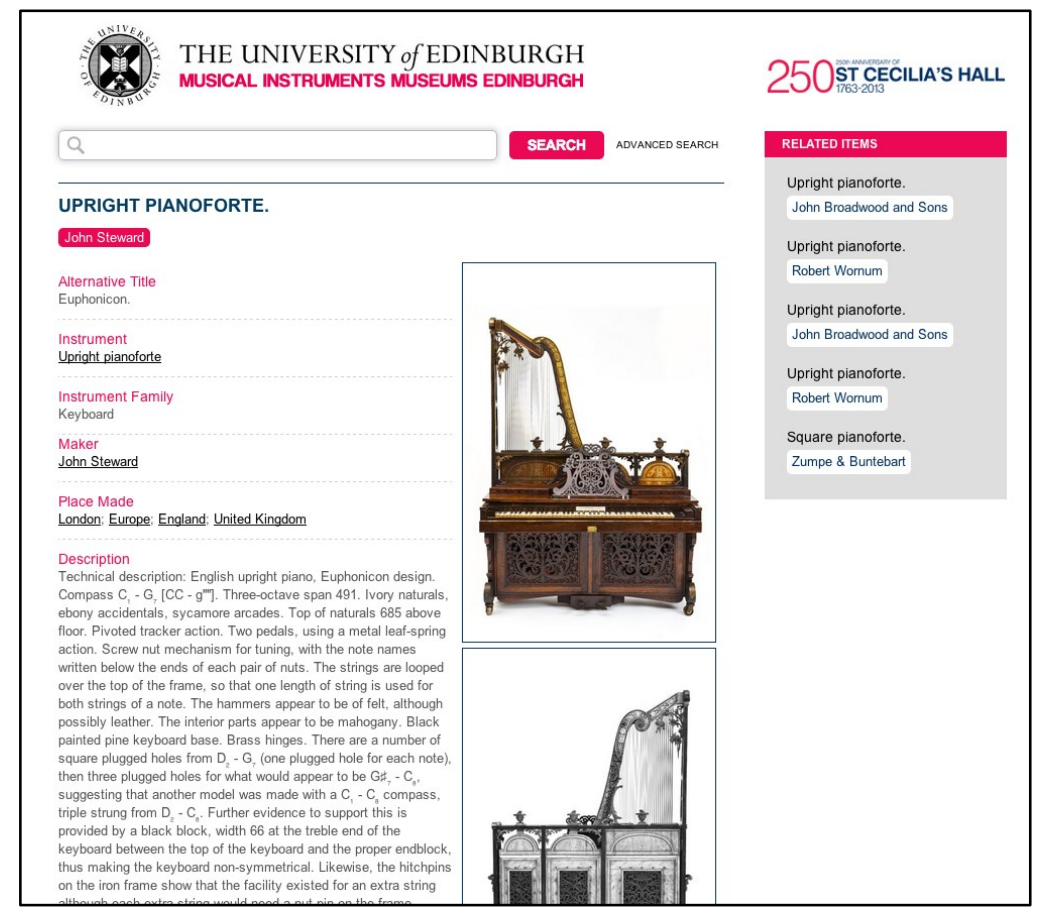

Figure 3: Item page for an upright pianoforte on collections.ed.ac.uk/mimed.

The musical instrument data is described in Vernon the Collections Management Software [18] in the SPECTRUM metadata standard; it is then exported and converted into Dublin Core for import into DSpace. The MIMO harvester aggregates the data in the LIDO format and it is then converted into the Europeana datamodel for 
inclusion in the Europeana portal. The original data creators are integral to the testing at each stage to ensure the data integrity following the data transformations.

Collections.ed.ac.uk/mimed is the public portal to the items catalogued in the Vernon CMS and harvested by MIMO. It allows L\&UC to showcase this internationally recognised collection and create its own online identity. It is the result of collaboration with external partners at MIMO and the University of Auckland as well as internal to L\&UC with the Curators, and Projects and Innovations. This model has been repeated with the Art Collection collections.ed.ac.uk/art and it is the intention to repeat this project model for the other rare and unique collections within L\&UC.

\section{CASE STUDY 2 COLLECTIONS MANAGEMENT (ARCHIVESSPACE)}

The Special Collections (manuscripts, archives and rare books) department at the University of Edinburgh, as with many long established Universities, inherited a variety of methods and approaches to capturing, describing, finding and accessing its collections. These ranged from $19^{\text {th }}$ century imposed systems translated throughout the $20^{\text {th }}$ century to project based cataloguing approaches in the early $21^{\text {st }}$ century. The array and diversity of these approaches, some based on antiquarian practice and bespoke methods of finding aids, some on older established library based methods, some developed uniquely by individuals within the University, meant that much of the ability to access the collections was based on the knowledge of individuals and experience of these systems. Time had to be taken to explain and practice the navigation through the systems with new members of staff and the collections were at times impenetrable for our users, both academic and the general public.

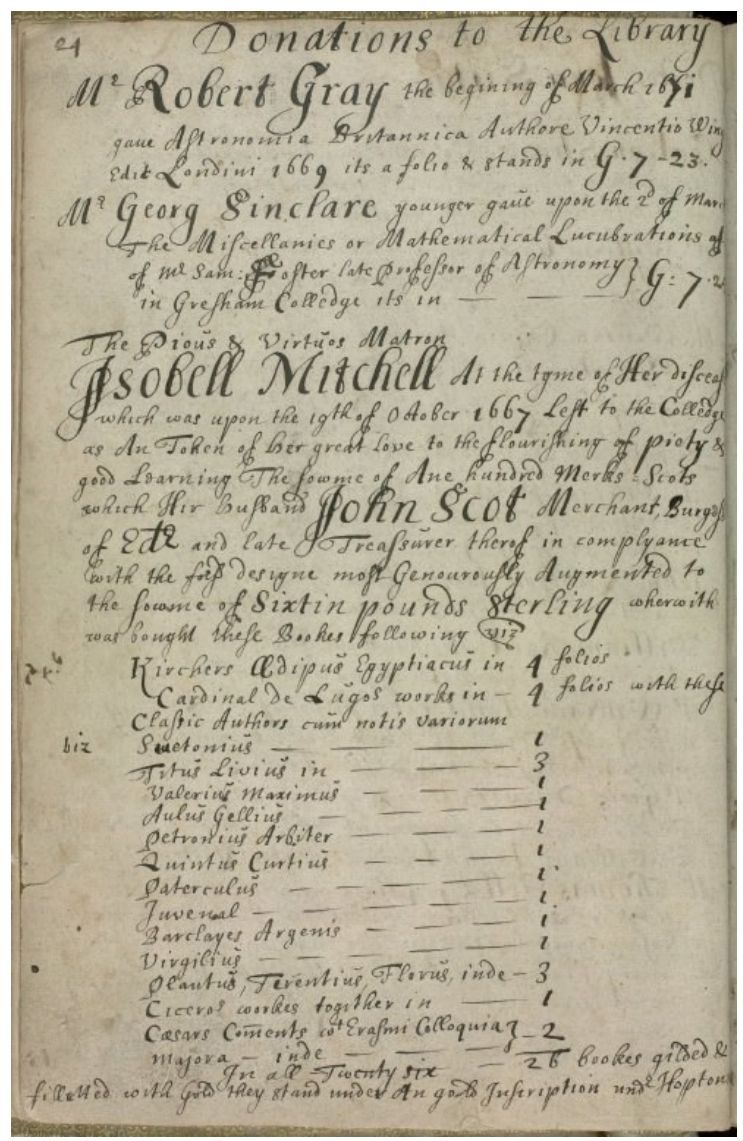

Figure 4: Entry from a donations book of the University of Edinburgh (1667-1824), showing early collections metadata. 
The changes in culture previously mentioned in this paper and the approach to provision of collections care, management and discoverability provided an opportunity to reflect, discuss and discover our practices and our requirements in terms of users, collections and skills in managing these kinds of collections.

\section{Legacy and Applying the Standards}

Much of the experience built up in the Special Collections department at the University of Edinburgh had been established through externally funded and collaborative cataloguing and preservation projects, such as the Research Support Libraries Programme projects NAHSTE [19] and GASHE [20], starting in the 1990s. This project based approach using testing, development and a variety of descriptive standards and methods still continues to allow focused work to take place on particular collections. Examples of this can be seen in the work done on the UNESCO recognised Carmichael Watson collections through an externally funded project [21], and others such as the Wellcome funded genetics archive s based Towards Dolly project [22] and Godfrey Thomson project [23] which linked and described data held within the University of Edinburgh and the University of Glasgow. Work on Encoded Archival Description, the use of authorities and subject indexing with function and activity descriptions had taken place in the early 2000s. Development of in house templates in XML for EAD required staff to be trained and fully up to speed with how this schema worked. With the team consisting of para professional staff, volunteers and interns, training in using the templates was time consuming and had to be repeated by staff with the expertise in the process and standards. The pioneering work through RSLP projects [24] had been an exemplar in managing intellectual access and applying international standards such as the International Standard of Archival Description (ISAD (G)) to increase the discoverability of certain collections which had been treated with less contextual, less relational and complex descriptive approaches.

Work on authority records using existing information but flexibly mapping it to ISAAR (CPF), NCA and other index based rules created a bank of authority records that were set up and ready for migration as required. An example of this was the harvesting of these records in the 2000s and packaging them correctly for inclusion in the UK wide Archives Hub portal for top level archival descriptions.

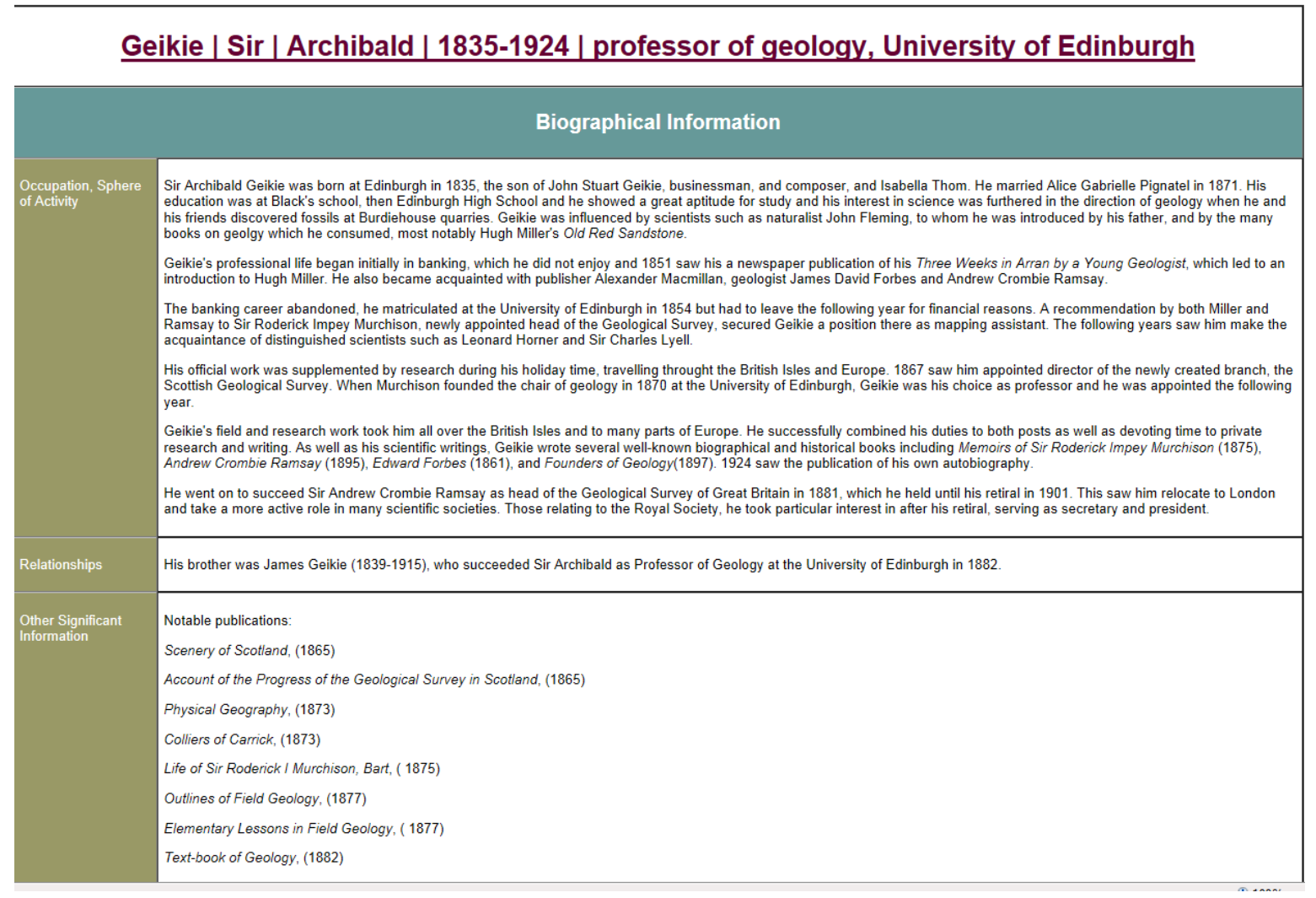

Figure 5: Authority record for Sir Archibald Geikie created through the NAHSTE project. 
The will to ensure the University had high quality professional, authoritative and user friendly descriptions of its unique and world renowned collections has been a constant since the first University Archivist was appointed in 1994. From the RSLP projects, ISAD (G) descriptions were posted and structured in a compliant friendly approach, which was intellectually rigorous in terms of archival practice and science. Clear hierarchical structures and investigations into contextual cataloguing, with some early functional and activity descriptions created through the GASHE project, were now available. There was professional standards mapping, checking of indexing and authority data with links to the collections descriptions. A fairly robust MySQL/PHP web-delivered collections management system had been built in-house by the Deputy University Archivist, but this even had its limitations despite being able to provide access to some of the collections detail and authority control. It could not however provide the functionality of describing collections in EAD directly

With no overall system managing the archival and manuscript collections, and while the project based approach was good for development, consistent method and system underpinning core archival work was still required. This system would need to ingest all the descriptive records, connect them, and map them to our other collections such as art, object, subject based or more general library collections.

\section{Change, Ambition and Vision}

With the discoverability of the collections still being limited, a more user friendly approach was still the ambition. The standards were present but often there were only top level (Fonds) descriptions, or if lower level description was present it was constructed with many layers and therefore made fairly complex. Some of this was through rigid application of the standards and an element of flexibility was required. It was recognised that treatment of different types of collection from corporate and institutional records to personal papers (with the inclusion of objects) and published work or academic research all had different requirements, users and contained different types of data.

The impact of Google as a search engine also played its role in how discoverable collections could be and the development of dynamic finding aids. This expanded the options and allowed the rethinking of what UoE collections needed and could do. Alongside this there was an expansion in the UK profession and the use and linking of technology and skills needed with thought being put to born digital records and how they and their metadata needed to be managed. Work has been done on analysing this growth in skills and awareness [25]. These provided a catalyst for the sharp rise in projects in the late 1990s and early 2000s with a focus on description and capturing metadata. Descriptive standards schema, such as EAD and EAC, had been developed and were becoming the normal practice. As more information on collections was being made available, this needed to be consistent and reliable for ease of access and understanding.

\section{Analysis of Systems and Requirements}

As a top UK University (17 ${ }^{\text {th }}$ in the QS World Rankings 2013/2014) the desire to have world renowned collections that reflect that status in research and learning was another influence on developing a world class catalogue. Investment had been made in the physical environment for the collections and needed to be reflected in the intellectual access to them.

Members of the special collections team had made improvements and built an in-house bespoke system to manage the metadata and collections descriptions, however this was not fully supported, difficult to maintain and reliant on one person. For an institutional service such as this, this was too high a risk as there was a single point of failure.

As the expertise was available in house, the requirement was not there to contract consultants to fill a skills gap. The gap identified was the scalability and cost effectiveness of maintaining an in house system that was able to span older arrangement and cataloguing systems as well as the new standard approaches. There was an urgent need for cross-collections cataloguing and management due to the upsurge in collections coming in and inability of previous systems to cope with demand, professional standards and tasks.

The timing for looking again at the system used came after redevelopments in the makeup of the teams and skills. Archival staff had followed developments [26] with systems such as ICA -ATOM and Archivists Toolkit that were being driven from the archives sector rather than commercial enterprise, to see if they would provide a 
potential solution for managing the collections data. In needing to develop a business case for funding and approval, analysis of these systems was undertaken.

Archivists Toolkit was tested before ArchivesSpace became the successor system and proved to be the contender due to the functionality and development opportunities offered. With the expertise held at the University and the diverse nature of the collections, there was a desire to be involved in the development and to influence a collections management system to suit the specific needs of the institution, while playing a constructive part in our professional communities. In 2013 Special Collections had around 6,000 accession records, about 1300 collections, with hundreds of lower-level descriptions beyond that. There was also a very conservative estimate of over 17,000 authority terms. These figures only show part of the picture. As previously described, this data was scattered around different systems and was very difficult to measure output in quantitative terms. The desire to bring all of this data together and link it was clear. ArchivesSpace allowed us to do this.

\section{Archivist's Toolkit to ArchivesSpace}

The need to review the status of the Archivist's Toolkit implementation coincided with the structural changes that occurred within Library and University Collections and led to a new project team being formed. The Projects and Innovations Team act as project managers with team members from across the disciplines within the section: archivists, software developers, IT support. The establishment of a team with a shared vision and values and common objective; to make the archival collections discoverable and easy to manage, resulted in a project with an appetite for change and momentum to move forward. An evaluation of Archivist Toolkit in the summer of 2013 corresponded with the deprecation of this software in favour of ArchivesSpace which brought it together with another US archive sector led system ARCHON. Our appraisal of an installation of a pre-release version of ArchivesSpace led us to the conclusion that it was a good match for our requirements and as users of other open source software that it would be a good fit within our suite of IT services. The project team then prepared a proposal to the Senior Management Team advocating that the University join as an ArchivesSpace member [27]. This proposal was accepted with the University becoming the first European member. Although it is provided as open source software and therefore can be used for free, the ArchivesSpace membership model allows users the opportunity to get access to systems support, members-only mailing lists, and participation in the setting of user, technical and services roadmaps.

In the past archive and special collections cataloguing had not been counted at lower levels and ArchivesSpace would make provision for this knowledge and control of what data we would have and the information about our collections (following on from the work begun in Archivists Toolkit). The flexibility in the system also meant that professionally we could look at which level is best to catalogue a collection to, within the timeframe available for projects.

What appealed about ArchivesSpace?

- The collections catalogues could become available in real time.

- Potential opportunities available as a result of collaborative working brought about by the L\&UC restructuring.

- Ability to meet the demand to make collections to be available, interoperable and for a long-term solution allowing projects to come into core collections management.

- A more streamlined and efficient method of cataloguing straight into a system; provides more meaningful training to allow an increase in the appropriate use of volunteers.

- A streamlined way for frontline reading room staff to find material and be self-sufficient in doing this.

- The open source nature provided us with a vehicle for cross-collections searching and quality consistent metadata for flexible use of collections in a sustainable way.

- It will allow us to respond more effectively to requests, events, projects and working closely with worldwide partners.

- Discovery and metadata will be managed in a system that it is possible to integrate with the wider Library IT infrastructure.

- An established support network, which have been working on defining the requirements and testing of the software. Software support via mailing lists, phone and email.

- Opportunities to develop and customise ArchivesSpace through its open source platform.

- Relatively low cost. 
No one else in the UK is currently involved in the professional collections management or developmental aspects of ArchivesSpace. It also appealed due to the opportunities for knowledge exchange as it was being developed in conjunction with well-resourced major US Universities and cultural/ historical bodies. We were also reassured that the involvement of members within the development of ArchivesSpace and building upon Archivist's Toolkit and Archon, many of the usual curatorial questions about the system meeting standards had been considered and addressed already.

From a technical perspective, ArchivesSpace is open source, and is built in reasonably ubiquitous and therefore reliable technologies. It utilises a MySQL database, SOLR indexing and it can be run under Tomcat or Jetty. The code is written in JRuby, with Bootstrap web clients. Rather than having to install lots of web clients for different users, four webapps provide the public access, admin area, SOLR search functionality, and backend REST API. The code is all available in GitHub https://github.com/hudmol/archivesspace .

The 'backend' keeps the bird's eye view on everything; the 'admin area' is where all the cataloguing and imports take place; the 'public site' is where an anonymous user can come in and search the archive at a reasonably detailed level. The SOLR webapp is essential for the population of the public site. This all means that the practice of creating information in a bespoke collections management tool whilst also generating EAD records in an XML editor is brought all together in one place.

We also needed to ensure it mapped to our known user needs and requirements. How did we know what they wanted? Rather than adopt the usual questionnaires which had been part of gathering our user data, we knew from consistent anecdotal experience that navigation through descriptive information was difficult. Users wanted to find the data about our collections, for it to be easily understandable and clear, and to find it easily again if they needed to search for it. An academic environment also requires the information to be accurate and authoritative and include references to further collections and information that was known about. This linked data needed to be easily navigable for users. We derived this information from project feedback, analysis of user activity and the nature of enquiries we received with a significant proportion reliant on special collections staff to undertake basic signposting to collections. The combination of this evidence provided us with real issues users faced while undertaking their research.

As well as the archivists and project team, the Library Digital Development Team has been heavily involved in the implementation of this project through the installation, configuration, and systems support during the loading of collections data. All of the cross discipline team have been involved together in the set-up of an internal mailing list for discussion, attendance at workshops for focused testing and data loads, and development of tools for data migration from the existing bespoke authorities database.

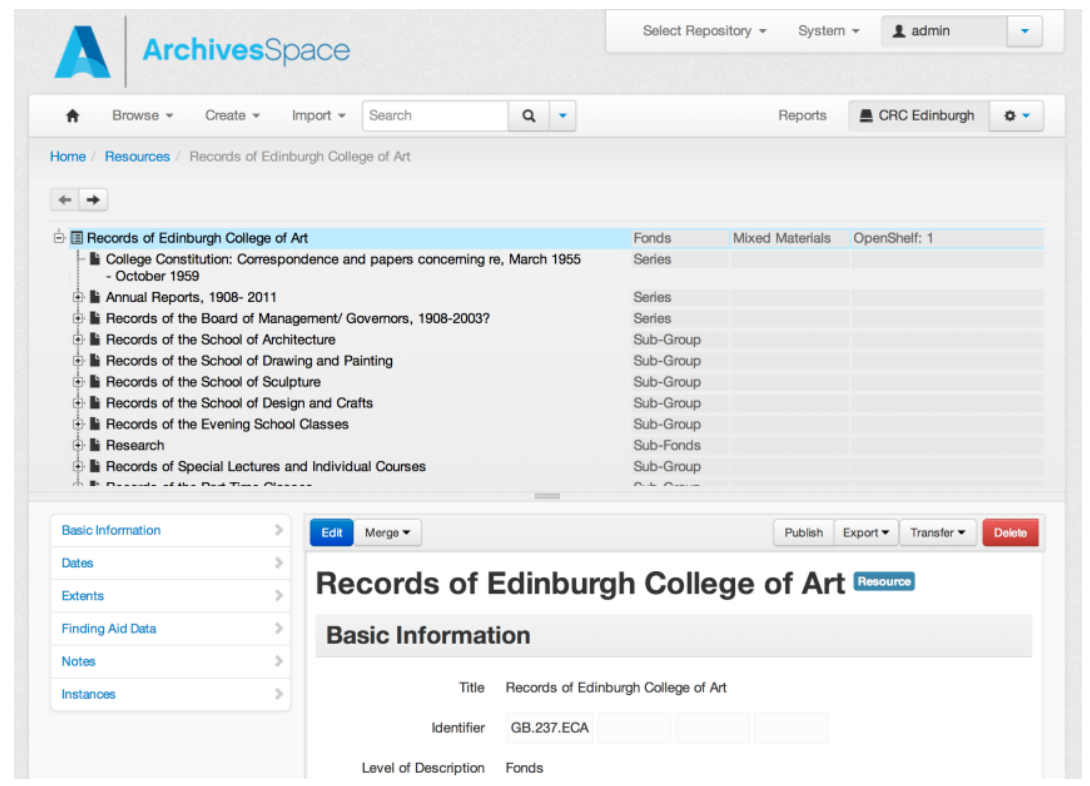

Figure 6: Resource description as seen in the administrative view of Archives Space for Edinburgh College of Art archives. 


\section{CONCLUSION}

In conclusion Library and University Collections at the University of Edinburgh has experienced a cultural shift in the way its data is linked, managed and made available. The ambition with these projects which has been embedded in working practice to showcase the world class collections that range from manuscripts detailing Scotland's role in international affairs, those that present literary and cultural icons such as Robert Burns and Sir Walter Scott, unsurpassed musical instrument collections to archival collections of scientific significance of genetic research and health and welfare developments is now a sustainable part of our work. This extends to linking these to library, archival, art and museum collections across the university and wider afield to make our data truly linked and interoperable. This has only been achievable through the combining of skills, standards, expertise, flexibility, and open-minded professionalism to create a working of culture of positive compromise. This culture of communication includes archivists and collections staff learning a little more of the 'dark' side of digital development work and systems, and the digital library developers learning about collections and the sensibility of the care for those collections and what we know about them. The overarching project management provided the core structure for project focus and movement to free up the creativity and technical skills of both these groups to work together to achieve platforms of authoritative linked data about the collections at the University of Edinburgh.

The next question we will be asking ourselves is how far this convergence will extend. There is soon to be a new library management system rolled out for library materials. Work has begun to map ArchivesSpace with this system and the staff from the projects mentioned in this paper have led on aspects of the metadata mapping. We hope that this will inform and standardise a library and university collections wide authorities database. Our digital image collections we also will map through this to provide more of a visual element to our catalogues. Through all this work we have learnt that all kinds of standards, skills and good practice can be used and inform conjointly with to allow for the mapping and interoperability. Future projects are extending this integration of data for the good of the collections discovery and ease of use for researchers. This includes a Wellcome funded project which will work with the National Library of Scotland on the archives and library of W. R. D. Fairbairn held across our two institutions making collection data about them linked and easily cross searchable.

\section{ACKNOWLEDGEMENTS}

The authors would like to thanks the Library and University Collections Senior Management Team members Dr John Scally, Stuart Lewis, Jackie MacBeath, Dr Joseph Marshall and Arnott Wilson for their support and help in writing this paper. We would also like to thanks colleagues Grant Buttars and Scott Renton for extra information which informed the process. Final thanks goes to project and development partners ArchivesSpace, Archives Hub, MIMO and University of Auckland Library Applications Development \& Support. 


\section{REFERENCES}

1. MIMO website http://www.mimo-international.com/MIMO/

2. Europeana website http://www.europeana.eu/

3. Archives Hub website http://archiveshub.ac.uk/

4. ArchivesSpace website http://www.archivesspace.org/

5. Archives Portal Europe website http://www.archivesportaleurope.net/

6. Staffing structure of Library and University Collections, University of Edinburgh. 2013

http://www.ed.ac.uk/polopoly_fs/1.17448!/fileManager/L\%26UC\%20ORG\%20CHART\%20JULY\%2014.pdf

7. Library and University Collections, University of Edinburgh. 'Vision Values, Strategic Goals and Key Performance Areas, 2013 - 2017'

http://www.docs.is.ed.ac.uk/docs/librarypapers/LAUC $\% 20$ Vision $\% 20$ Values $\% 20$ and $\% 20$ KPAs $\% 20$ Final $\% 2018 \% 2012 \% 2013$.pdf

8. University of Edinburgh Strategic Plan http://www.docs.sasg.ed.ac.uk/gasp/strategicplanning/201216/StrategicPlan201216.pdf

9. MIMO documentation on vocabulary standards and toolkit http://www.mimo-international.com/vocabulary.html http://www.mimo-international.com/toolkit.html

10. St Cecilia's hall project website http://www.stcecilias.ed.ac.uk/ DSpace website http://www.dspace.org/

Presentation on DSpace http://figshare.com/articles/Reusing_repository_technology_for_Cultural_Heritage_and_Special_Collections/1 $\underline{089651}$

11. Theses Alive Project https://www.era.lib.ed.ac.uk/handle/1842/423

12. Open Access, open source and e-theses: the development of the Edinburgh Research Archive https://www.era.lib.ed.ac.uk/handle/1842/811

13. Repository Review documentation http://www.docs.is.ed.ac.uk/docs/Library-Collections/Repository\%20Review\%20Infrastructure.pdf

14. DSpace Discovery https://www.youtube.com/watch?v=abRSXTUEwws Apache SOLR http://lucene.apache.org/solr/

15. Skylight development and technical documentation https://github.com/skylightui/skylight

16. Repository as a Service (RaaS) K. Shepherd, S. Lewis, Y. Latt, A. Schweer, A. Field, University of Auckland, Journal of Digital Information 13(1) Article number 32012 https://researchspace.auckland.ac.nz/handle/2292/17764

17. University of Edinburgh branding and logo guidelines http://www.ed.ac.uk/polopoly fs/1.16489! fileManager/university-of-edinburgh-logo-guide.pdf

18. Vernon Collections Management System website http://www.vernonsystems.com/

19. Navigational Aids for the History of Science and Technology website http://www.nahste.ac.uk/project/

20. Gateway to Archives of Higher Education website http://www.gashe.ac.uk/

21. Carmichael Watson Archive Project website http://www.carmichaelwatson.lib.ed.ac.uk/cwatson/

22. Towards Dolly: Edinburgh, Roslin and the Birth of Modern Genetics website http://www.archives.lib.ed.ac.uk/towardsdolly/

23. http://libraryblogs.is.ed.ac.uk/godfreythomsonproject/

24. The RSLP Collection Description Schema, P. Johnston (2003) http://www.ukoln.ac.uk/cd-focus/briefings/bp2/bp2.pdf

25. National Archives (UK) research reports including those on workforce and online access http://www.nationalarchives.gov.uk/archives-sector/research-reports.htm

26. Archival Management Software: A Report for the Council on Library and Information Resources, L. Spiro, January 2009 http://www.clir.org/pubs/reports/spiro/spiro_Jan13.pdf

ArchivesSpace: A Next-Generation Archives Management System M. A. Matienzo, K.Kott, (Museums and the Web conference 2013)

http://mw2013.museumsandtheweb.com/paper/archivesspace-a-next-generation-archives-management-system/

27. ArchivesSpace members brochure and membership pages

http://www.archivesspace.org/sites/default/files/ArchivesSpace-Inline-Brochure.pdf 